\title{
MINIMALLY OVERLAPPING PATHS SETS FOR CLOSED CONTOUR EXTRACTION
}

\author{
Julien Mille ${ }^{1}$, Sébastien Bougleux ${ }^{2}$ and Laurent Cohen $^{3}$ \\ ${ }^{1}$ Université de Lyon, CNRS \\ Université Lyon 1, LIRIS, UMR5202, F-69622, Villeurbanne, France \\ ${ }^{2}$ Université de Caen Basse-Normandie, CNRS \\ GREYC, UMR6072, F-14050, Caen, France \\ ${ }^{3}$ Université Paris-Dauphine, CNRS \\ CEREMADE, UMR7534, F-75016, Paris, France \\ julien.mille@liris.cnrs.fr \\ sebastien.bougleux@unicaen.fr \\ coheneceremade.dauphine.fr
}

Keywords: Segmentation, boundary extraction, minimal path, active contour, overlap

\begin{abstract}
Active contours and minimal paths have been extensively studied theoretical tools for image segmentation. The recent geodesically linked active contour model, which basically consists in a set of vertices connected by paths of minimal cost, blend the benefits of both concepts. This makes up a closed piecewise-defined curve, over which an edge or region energy functional can be formulated. As an important shortcoming, the geodesically linked active contour model in its initial formulation does not guarantee to represent a simple curve, consistent with respect to the purpose of segmentation. In this paper, we propose to extract a similarly piecewise-defined curve from a set of possible paths, such that the resulting structure is guaranteed to represent a relevant closed curve. Toward this goal, we introduce a global constraint penalizing excessive overlap between paths. ${ }^{a}$
\end{abstract}

\footnotetext{
${ }^{a}$ This work was supported by a grant from the GdR ISIS (french research group on Information, Signal, Image and Vision) as well as the REVES project (funded by the French National Agency for Research with the reference ANR-10-CORD-005)
}

\section{Introduction}

Methods addressing the problem of two-phase segmentation based on energy minimization techniques and variational principles provide a solid mathematical background, and have proven to find suitable solutions in many practical situations. Among them, active contour models consist in deforming an initial curve until it captures the boundary of the target object. Whether they are implemented in an explicit fashion or using level sets, their evolution is usually driven by gradient descent of the Euler-Lagrange equation, which makes them sensitive to local minima, specifically in the presence of noisy images. Consequently, the quality of the resulting segmentation strongly depends on the initial contour position. Several attempts have been made to reduce this sensitivity, including the addition of terms such as the balloon force (Cohen, 1991) or the use of discrete optimization heuristics such as dynamic programming (Amini et al., 1990) or greedy algorithms (Williams and Shah, 1992). However, these methods still lead to a local minimum of the energy.

To overcome sensitivity to local minima, (Cohen and Kimmel, 1997) proposed to find a global minimum of the geodesic active contour functional, provided that one or two points of the target object boundary are initially supplied by the user. The resulting global geodesic curve, which can be respectively closed or open, is efficiently derived from the solution of the Eikonal equation obtained with the Fast Marching method (Tsitsiklis, 1995; 
Sethian, 1996). Since the control points are fixed and must be located on the target contour, this latter model does not represent a curve which deforms its shape. Moreover, due to the restricted number of these points, the geodesic may fail to capture a relevant contour if the image is too noisy, not enough contrasted, or if the target contour is too lengthy. While several methods concentrate on avoiding this second drawback (Benmansour and Cohen, 2009) (Benmansour and Cohen, 2011) (Kaul et al., 2010), the geodesically linked active contour model of (Mille and Cohen, 2009) allows to overcome the first one. This latter model combines the advantages of geodesics with the ones of greedy algorithms in order to deform a piecewise geodesic curve. Moreover, it is also able to include region-based energies, such as the minimal variance term proposed by (Chan and Vese, 2001), or even shape prior terms. Whereas this model is relatively robust to local minima, it can fail to construct a valid closed curve, from the initialization step to the end of the evolution.

To overcome this drawback, we design a new energy functional allowing to find a piecewise smooth curve with minimal overlapping. Given several possible relevant paths, subsequently referred to as admissible paths, the key idea of our contribution is to select the combination of paths generating the most relevant contour. In this extent, we introduce an energy functional, combining contour and region terms with a novel overlapping measure.The construction of admissible paths, the overlapping energy as well as the selection of the optimal combination of paths are described in Section 3. The effectiveness of our extended geodesically linked active contour model, whether given initial points are located on the target contour or far from it, is shown in Section 4 through several experiments. The concepts on which relies the proposed approach are recalled in the following section.

\section{Related concepts}

\subsection{Minimal paths}

To extract structures in a given image $I: \mathcal{D} \rightarrow \mathbb{R}^{d}$, (Cohen and Kimmel, 1997) proposed to find curves of minimal length according to an heterogeneous isotropic metric defined from a potential $P: \mathcal{D} \rightarrow \mathbb{R}^{*+}$. This potential, which is chosen to take lower values on the structure of interest, allows to measure the length of piecewise smooth curves $\gamma:[0,1] \rightarrow \mathcal{D}$ as fol- lows:

$$
L[\gamma]=\int_{0}^{1} P(\gamma(u))\left\|\gamma^{\prime}(u)\right\| \mathrm{d} u .
$$

In the context of contour extraction, curves should be located along edges. The potential is thus defined as $P=g+w$, where $g: \mathcal{D} \rightarrow \mathbb{R}^{+}$is a decreasing function of the gradient magnitude of the image (usually convolved with the derivatives of a Gaussian with given standard deviation $\sigma$ ),

$$
g(\mathbf{x})=\frac{1}{1+\left\|\nabla\left(K_{\sigma} * I\right)(\mathbf{x})\right\|}
$$

and $w \in \mathbb{R}^{*+}$ is a regularizing constant. The target image structure is then extracted by finding a path of minimal length among all paths connecting two given points $\mathbf{x}_{1}$ and $\mathbf{x}_{2}$ located on the structure

$$
\underset{\gamma \subset \mathcal{D}}{\operatorname{argmin}}\{L[\gamma]\} \quad \text { s.t. }\left\{\begin{array}{l}
\gamma(0)=\mathbf{x}_{1} \\
\gamma(1)=\mathbf{x}_{2}
\end{array} .\right.
$$

Such a globally defined minimal path is called a geodesic. The solution of minimization problem (3) can be obtained by considering the geodesic distance map, also referred to as the minimal action map, $U_{\mathbf{v}}: \mathcal{D} \rightarrow \mathbb{R}^{+}$which assigns, to each point $\mathbf{x} \in \mathcal{D}$, the length of the minimal path connecting $\mathbf{x}$ to a given point $\mathbf{v} \in \mathcal{D}$ :

$$
U_{\mathbf{v}}(\mathbf{x})=\inf _{\gamma}\{L[\gamma]\} \text { s.t. }\left\{\begin{array}{l}
\gamma(0)=\mathbf{v}, \\
\gamma(1)=\mathbf{x} .
\end{array}\right.
$$

This map is the unique viscosity solution of the Eikonal equation

$$
\left\{\begin{array}{l}
\left\|\nabla U_{\mathbf{v}}(\mathbf{x})\right\|=P(\mathbf{x}), \quad \forall \mathbf{x} \in \mathcal{D} \backslash\{\mathbf{v}\}, \\
U_{\mathbf{v}}(\mathbf{v})=0,
\end{array}\right.
$$

see for instance (Crandall et al., 1992). This allows to replace optimization problem (4) by a partial differential equation. Its discrete version, on a cartesian grid, can be efficiently solved by the Fast Marching (FM) method in $O(N \log N)$ operations, where $N$ is the number of grid points (Tsitsiklis, 1995; Sethian, 1996; Sethian, 1999). Once the distance map has been numerically computed, the minimal path between $\mathbf{v}$ and any other point $\mathbf{x}$ of $\mathcal{D}$ can be extracted by a gradient descent on $U_{\mathbf{v}}$

$$
\left\{\begin{array}{l}
\gamma^{\prime}(u)=-\frac{\nabla U_{\mathbf{v}}(\gamma(u))}{\left\|\nabla U_{\mathbf{v}}(\gamma(u))\right\|}, \\
\gamma(0)=\mathbf{x}
\end{array}\right.
$$

that corresponds to a back-propagation starting from $\mathbf{x}$ until $\mathbf{v}$ is reached. In practice, since the FM is a monotonically front propagation method, finding the minimal path bewteen two points does not require to compute the distance on the whole 


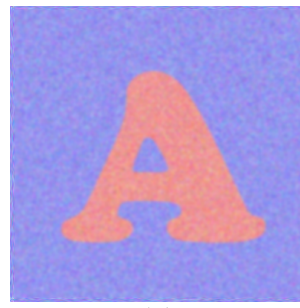

(a)

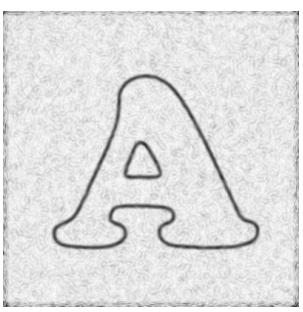

(b)

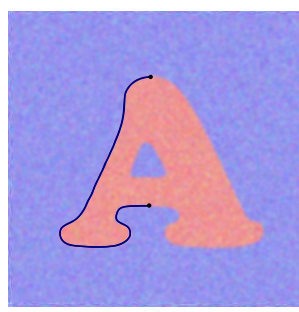

(c)

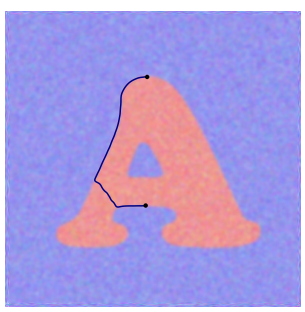

(d)

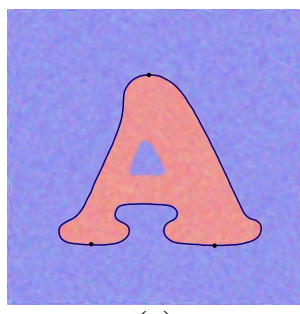

(e)

Figure 1: (a) Input image. (b) Potential $P$. (c) Geodesic between two given points. (d) Undesirable sructure extraction. (e) Piecewise geodesic curve.

domain $\mathcal{D}$. Starting from one point, the FM can be stopped when the second point is reached, ensuring that the minimal path can be extracted with (6).

The minimal path approach is not restricted to extract an open curve, provided its endpoints. In particular, in the context of object extraction, it is able to find a closed curve, provided only one point on the target object boundary. The closed curve is obtained by detecting a saddle point of the distance map and then by performing two back-propagations, in opposite directions, starting from this saddle point (Cohen and Kimmel, 1997). Whether the curve is closed or open, the minimal path approach can fail to extract the desired curve. As depicted in Fig. 1(d), some portions of the minimal path do not follow the desired curve. This happens for instance when $P$ is too noisy or not enough contrasted, when the length of the target curve is too important, or when the regularization constant $w$ is too high. This undesirable behaviour hides a sampling problem, that is one or two points are usually not enough to capture the whole desired curve.

To overcome this drawback, several approaches aim at finding a piecewise geodesic curve $\Gamma=\left(V,\left\{\gamma_{i}\right\}_{i}\right)$, where $V=\left\{\mathbf{v}_{i}\right\}_{i}$ is a set of vertices that samples the structure to extract, and $\left\{\gamma_{i}\right\}_{i}$ is the set of geodesics connecting pairs of succesive vertices (see Fig. 1(e)):

$$
\gamma_{i}=\underset{\gamma}{\operatorname{argmin}}\{L[\gamma]\} \text { s.t. }\left\{\begin{array}{l}
\gamma(0)=v_{i}, \\
\gamma(1)=v_{i+1} .
\end{array}\right.
$$

Given an initial vertex set, new vertices can be recursively and efficiently detected in several practical situations such that the resulting piecewise geodesic curve matches the desired structure. See (Benmansour and Cohen, 2009; Peyré et al., 2010) for a complete survey. In Section 3 we propose an alternative approach to overcome this drawback when a closed curve needs to be extracted. This approach is closely related to the geodesically linked active contour de- scribed in the following section.

\subsection{The geodesically linked active contour model}

In order to extract an object from an image, (Mille and Cohen, 2009) proposed an active contour model explicitely represented by a closed piecewise geodesic curve, allowing initialization inside the object or around the object boundary. The optimal contour is defined as the piecewise geodesic closed curve $\Gamma=\left(V,\left\{\gamma_{i}\right\}_{1 \leq i \leq n}\right)$ that minimizes a weighted sum of edge-based and region-based energy functionals:

$$
E[\Gamma]=\omega_{\text {edge }} E_{\text {edge }}[\Gamma]+\omega_{\text {region }} E_{\text {region }}[\Gamma] .
$$

The edge-based energy integrates the edge indicator function $g$ (Eq. (2)) along the geodesics

$$
E_{\text {edge }}(\Gamma)=\frac{1}{|\Gamma|} \sum_{i=1}^{n} \int_{0}^{1} g\left(\gamma_{i}(u)\right)\left\|\gamma_{i}^{\prime}(u)\right\| \mathrm{d} u .
$$

In order not to penalize lengthy contours, it is normalized by the euclidean length

$$
|\Gamma|=\sum_{i=1}^{n} \int_{0}^{1}\left\|\gamma_{i}^{\prime}(u)\right\| \mathrm{d} u .
$$

One may note that the edge indicator $g$ is used instead of the potential $P$ so that the euclidean component of the curve length is not taken into account. This ensures that short curves, which could be undesirable shortcuts, are not preferred over longer ones. This edge term may be associated with a balloon term similar to the one introduced in (Cohen, 1991) in order to increase the capture range of the contour when located far from the object boundary.

In addition, the region-based energy allows to overcome limitations of the edge-based only model, in particular when dealing with noisy, low-constrasted or textured images. (Mille and Cohen, 2009) proposed to use a modified version of the two-phase piecewise constant segmentation model developped 

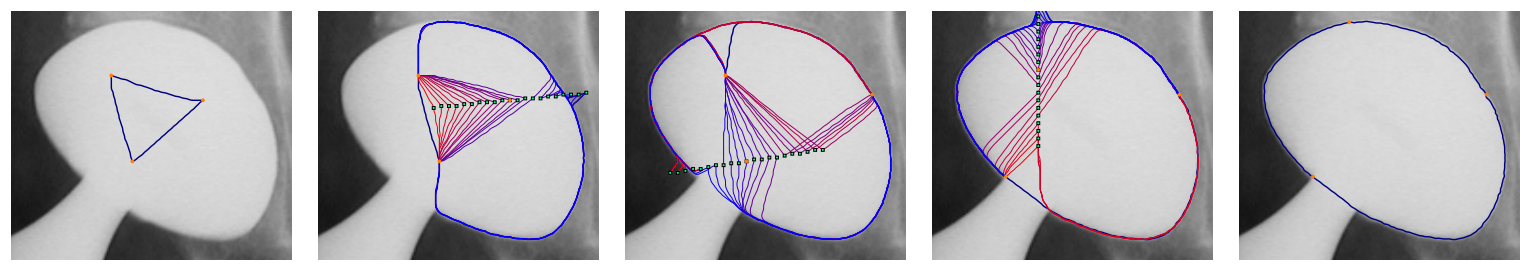

Figure 2: Evolution of geodesically linked active contour: in the evolution steps, all test geodesics from neighboring vertices to test positions in windows are represented.

by (Chan and Vese, 2001). Assuming that curve $\Gamma$ partitions the image into inner region $\Omega_{\text {in }}$ and outer region $\Omega_{\text {out }}$, the region term is expressed as the sum of inner and outer image variances:

$$
\begin{aligned}
E_{\text {region }}[\Gamma]= & \frac{1}{\left|\Omega_{\text {in }}\right|} \int_{\Omega_{\text {in }}}\left\|I(\mathbf{x})-\mu_{\text {in }}\right\|^{2} \mathrm{~d} \mathbf{x} \\
& +\frac{1}{\left|\Omega_{\text {out }}\right|} \int_{\Omega_{\text {out }}}\left\|I(\mathbf{x})-\mu_{\text {out }}\right\|^{2} \mathrm{~d} \mathbf{x},
\end{aligned}
$$

where $\mu_{\text {in }}$ and $\mu_{\text {out }}$ are average colors in these regions. Following (Mille, 2009), a relaxed image homogeneity term focusing on the vicinity of the curve, referred to as narrow band region term, was also addressed as a possible replacement for the previous region term.

Evolution of active contours, whether they are implemented explicitly or in a level-set fashion, is usually performed with gradient descent of the EulerLagrange equation. However, in the present case, the energy cannot be differentiated with respect to a given vertex $\mathbf{v}_{i}$. It depends on geodesics linked to $\mathbf{v}_{i}$, which are not expressed in closed form. Hence, the piecewise geodesic structure is evolved thanks to a greedy algorithm similar in principle to the one proposed in (Williams and Shah, 1992), which is discrete by nature and does not imply differentiation. Basically, vertices are moved in local windows in order to minimize the selected energy. Let $\mathcal{W}_{\mathrm{N}}$ be a normaloriented window of length $m$ centered at vertex $\mathbf{v}_{i}$ :

$$
\mathcal{W}_{\mathrm{N}}\left(\mathbf{v}_{i}\right)=\left\{\mathbf{v}_{i}+k \mathbf{n}_{i} \mid k=-\frac{m}{2} \cdots \frac{m}{2}\right\}
$$

where $\mathbf{n}_{i}$ is the inward unit normal vector, estimated by finite difference on corresponding points on geodesics $\gamma_{i}$ and $\gamma_{i+1}$, respectively. Greedy evolution is performed by moving vertex $\mathbf{v}_{i}$ to the position in the window which corresponding geodesically linked contour has the smallest energy $E$. Let us consider a test position $\tilde{\mathbf{v}}_{i}$ belonging to the window, and its associated test geodesics $\tilde{\gamma}_{i-1}$ and $\tilde{\gamma}_{i}$ linking it to the neighbors of $\mathbf{v}_{i-1}$ and $\mathbf{v}_{i+1}$, respectively. The evolution scheme for vertex $\mathbf{v}_{i}$ is formalized by the iteration:

$$
\mathbf{v}_{i}^{(t+1)}=\underset{\tilde{\mathbf{v}}_{i} \in \mathcal{W}_{\mathrm{N}}\left(\mathbf{v}_{i}^{(t)}\right)}{\operatorname{argmin}} E(\tilde{\Gamma})
$$

where $\tilde{\Gamma}$ is the tested piecewise geodesic curve:

$$
\tilde{\Gamma}=\left\{\gamma_{1}, \ldots, \gamma_{i-2}, \tilde{\gamma}_{i-1}, \tilde{\gamma}_{i}, \gamma_{i+1}, \ldots, \gamma_{n}\right\}
$$

The behavior of the geodesically linked active contour is depicted in Fig. 2. We can observe that it is able to capture accurately the object boundaries with a reduced number of vertices.

While the geodesically linked active contour model allows to blend the benefits of minimal paths and region-based terms, it turns out to have a significant drawback, as its initial state is not necessarily a simple closed curve. As depicted in Fig. 3(a), this can occur when the initial vertices are unevenly distributed around the target boundary. In this case, geodesics are very likely to gather on particular sides of the target instead of roughly covering the boundary. The reason is that each geodesic is generated independently of the others, such that the obtained piecewisegeodesic curve does not depend on the visiting order of pairs of adjacent vertices. This undesirable phenomenon may occur either as soon as the geodesically linked contour is initialized, or after several evolution steps on a previously well initialized contour.

As in Section 2.1, this problem can be seen as a sampling one. Intuitively, one could think of imposing evenly spaced vertices, as depicted in Fig. 3(b), or adding vertices near the parts of the target boundary which are not covered by the piecewise geodesic curve, like in Fig. 3(c). In the considered context, such sampling criteria are difficult to express, since the target boundary is unknown and applications usually need minimal user interaction. Otherwise, one could think of imposing hard constraints on the overlapping between paths or penalizing paths enclosing a region with excessively small area, but the independent construction of paths prevents such constraints to be implemented. We address this shortcoming in what follows.

\section{Finding the best path set}

To overcome the drawbacks of the geodesically linked active contour model, we focus on determin- 


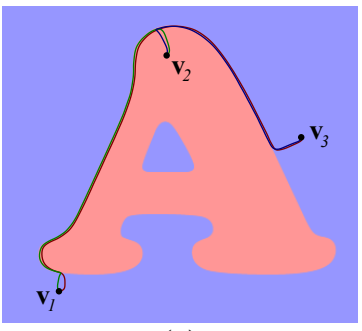

(a)

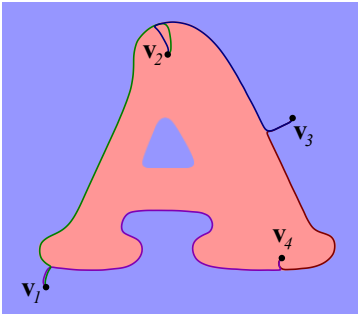

(c)

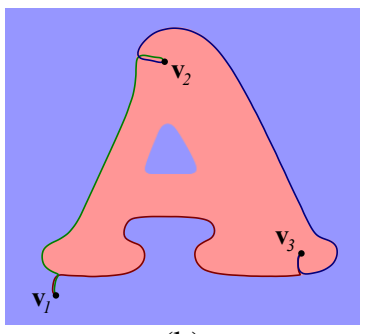

(b)

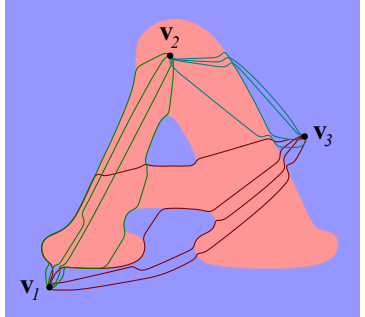

(d)
Figure 3: Towards a relevant initialization of the geodesically linked active contour model: (a) undesirable overlapping with unevenly spaced vertices, (b) improvement by even spacing of vertices, (c) improvement by addition of vertex, (d) admissible paths sets between pairs of vertices with $K=4$ paths per pair and high regularization constant $w$

ing a more relevant contour representation which preserves the advantages of piecewise geodesic curves. Assuming that several possible relevant paths linking successive vertices are available, the idea of our contribution is to select the combination of paths generating the most relevant boundary curve. This piecewise smooth closed curve is built by selecting a single path from each set, related to each pair of successive vertices. The relevancy of the generated contour is measured by an energy functional, combining contour and region terms with an overlapping measure. This last term ensures the resulting curve to minimally overlap.

\subsection{Sets of admissible paths}

Let $V=\left\{\mathbf{v}_{i}\right\}_{1 \leq i \leq n}$ be a sequence of $n$ given vertices. Instead of a single geodesic $\gamma_{i}$ for each pair of successive vertices $\mathbf{v}_{i}$ and $\mathbf{v}_{i+1}$, we consider a set $S_{i}$ of $K$ admissible paths available for this pair, as exemplified in Fig. 3(d):

$$
S_{i}=\left\{\gamma_{i, j}\right\}_{1 \leq j \leq K}
$$

Paths in $S_{i}$ are sorted by cost in ascending order, so that $\gamma_{i, 1}$ actually corresponds to the minimal path between $\mathbf{v}_{i}$ and $\mathbf{v}_{i+1}$ whereas the remaining curves $\gamma_{i, j}$, $2 \leq j \leq K$, are only short paths of increasing cost. Moreover, all paths in $S_{i}$ are constrained to be pairwise disjoint, except at their endpoints, which is for- mulated as the following condition:

$$
\begin{array}{ll}
\gamma_{i, j_{1}}(u) \neq \gamma_{i, j_{2}}(v), \quad \forall\left(j_{1}, j_{2}\right) \in[1 . . K]^{2}, j_{1} \neq j_{2}, & \forall(u, v) \in] 0,1\left[^{2}, u \neq v .\right.
\end{array}
$$

One may notice that the current approach is more constrained than the so-called $K$ shortest paths problem (Yen, 1971; Eppstein, 1998), which, in its basic formulation, does not impose paths to be disjoint. In the present case, the non-overlap constraint simplifies the generation of several paths. Intuitively, in a graph, the disjoint paths between a pair of vertices can be found by running several instances of the shortest path algorithm, after removal of vertices and incident edges belonging to already found paths.

In our approach, recall that paths are extracted using the Fast Marching method. Hence, the $K$ admissible paths are built by successive deletion of already existing paths from the potential map. Curve $\gamma_{i, 1}$ is the minimal path between $\mathbf{v}_{i}$ and $\mathbf{v}_{i+1}$ in the space endowed by the initial potential $P_{1}=P$. Once the minimal path $\gamma_{i, 1}$ has been computed, the second admissible path $\gamma_{i, 2}$ is sought under the constraint that it should not pass through points belonging to $\gamma_{i, 1}$. Hence, $\gamma_{i, 2}$ is not a geodesic in the space induced by potential $P$, but in the space induced by a modified potential $P_{2}$. The deletion of $\gamma_{i, 1}$ in the modified potential map is achieved by setting the potential to $+\infty$ at all points of the geodesic. Extending this principle to the construction of the $j^{\text {th }}$ admissible path $\gamma_{i, j}$ as shown in Fig. 4, a recursive definition of potential functions can be written as:

$$
P_{j}(\mathbf{x})= \begin{cases}+\infty & \text { if } \mathbf{x} \in \gamma_{i, j-1} \\ P_{j-1}(\mathbf{x}) & \text { otherwise. }\end{cases}
$$

This leads to the following recursive definition of the set of admissible paths:

$$
\begin{gathered}
\gamma_{i, j}=\underset{\gamma}{\operatorname{argmin}}\left\{\int_{0}^{1} P_{j}(\gamma(u))\left\|\gamma^{\prime}(u)\right\| \mathrm{d} u\right\} \\
\text { s.t. } \gamma_{i, j}(0)=\mathbf{v}_{i} \text { and } \gamma_{i, j}(1)=\mathbf{v}_{i+1} .
\end{gathered}
$$

From a practical point of view, one may note that the gradient descent scheme used to built path is continuous, which generates path points with real coordinates, whereas the potential is implemented on a discrete grid. Hence, in order to set $P_{j}(\mathbf{x})$ to $+\infty$, we actually set the 4 integer points surrounding $\mathbf{x}$ to $+\infty$.

\subsection{Paths configuration of minimal cost}

The computation of an admissible closed contour consists in selecting one path out of each set $S_{i}$, such that the contour resulting from the concatenation of selected paths exhibit desirable properties in the image. 

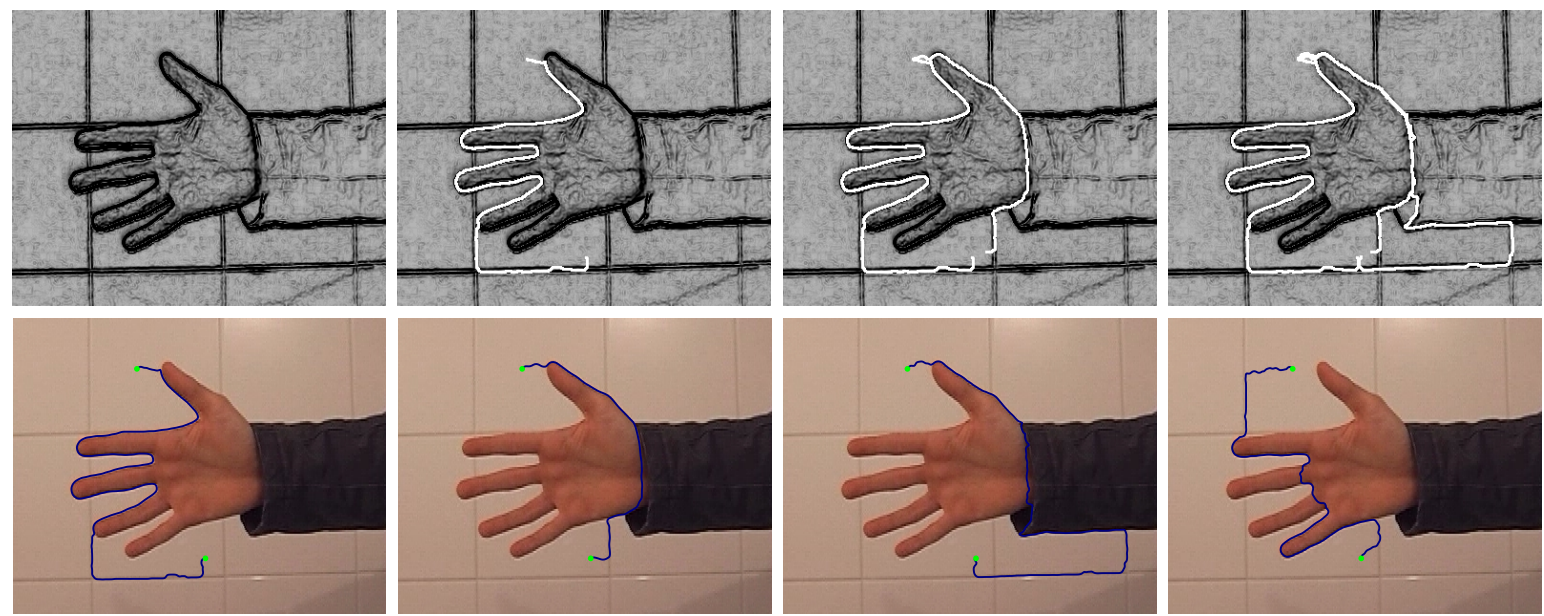

Figure 4: Successive potential maps $P_{j}$ (top row) and corresponding admissible paths $\gamma_{\cdot, j}$ (bottom row) given two endpoints.

One of these properties is that the generated contour should be simple, i.e. non-intersecting. In practice, it is reasonable to allow some overlapping between paths. A natural example arises when vertices are located far from the target boundaries, which might cause several admissible paths to have common sections before splitting up. Hence, the non-overlapping condition should be reformulated as a soft constraint. Towards this purpose, we first introduce the overlap measure $O$ between two curves:

$$
\begin{array}{r}
O\left[\mathcal{C}_{1}, \mathcal{C}_{2}\right]=\max \left(\frac{1}{\left|\mathcal{C}_{1}\right|} \int_{0}^{1} \psi\left[\mathcal{C}_{1}(u), \mathcal{C}_{2}\right]\left\|\mathcal{C}_{1}^{\prime}\right\| \mathrm{d} u,\right. \\
\left.\frac{1}{\left|\mathcal{C}_{2}\right|} \int_{0}^{1} \psi\left[\mathcal{C}_{2}(u), \mathcal{C}_{1}\right]\left\|\mathcal{C}_{2}^{\prime}\right\| \mathrm{d} u\right) .
\end{array}
$$

It may be considered as the similarity counterpart of the modified Hausdorff distance (Dubuisson and Jain, 1994), as the integrated quantity is a proximity measure instead of a distance. Penalty functional $\psi$ measures the cost of the proximity of point $\mathbf{x}$ to curve $\mathcal{C}$. We chose a truncated linear decreasing function of the euclidean distance between $\mathbf{x}$ and its nearest point located on $C$ :

$$
\psi[\mathbf{x}, \mathcal{C}]=\max \left(0,1-\alpha \min _{v \in[0,1]}\{\|\mathbf{x}-\mathcal{C}(v)\|\}\right),
$$

where weight $\alpha$ controls the decreasing slope, which is related to the fuzziness of the overlap cost. Note that $O$ is symmetrical and $O[\mathcal{C}, \mathcal{C}]=1$.

The computation of an admissible closed contour can be formulated as determining the sequence of labels $\left\{x_{1}, x_{2}, \ldots, x_{n}\right\} \in[1 . . K]^{n}$ minimizing an energy functional $E$, where label $x_{i}$ corresponds to the chosen path in set $S_{i}$ :

$$
\min _{\left\{x_{1}, x_{2}, \cdots, x_{n}\right\} \in[1 . . K]^{n}} E\left[\Gamma\left(\gamma_{1, x_{1}}, \gamma_{2, x_{2}}, \cdots, \gamma_{n, x_{n}}\right)\right],
$$

where $\Gamma\left(\gamma_{1, x_{1}}, \gamma_{2, x_{2}}, \ldots, \gamma_{n, x_{n}}\right)$ is the closed contour built by concatenation of paths $\gamma_{i, x_{i}}$. It is subsequently shortened to $\Gamma$ for simplicity. Energy $E$ is the mathematical formulation of required properties of $\Gamma$ within the image, extending the energy functional (8) involved in the geodesically linked active contour model. It is designed to penalize contours exhibiting strongly overlapping sections, poorly fitting to image edges or enclosing regions with high color disparity:

$$
\begin{aligned}
E[\Gamma]= & E_{\text {overlap }}[\Gamma]+\omega_{\text {edge }} E_{\text {edge }}[\Gamma] \\
& +\omega_{\text {region }} E_{\text {region }}[\Gamma] .
\end{aligned}
$$

Weights $\omega_{\text {edge }}$ and $\omega_{\text {region }}$ are user-defined parameters controlling the relative significance of the edge and region terms over the overlap term. This last one is defined by applying the overlap measure defined in equation (12) over all pairs of paths:

$$
E_{\text {overlap }}[\Gamma]=\sum_{i=1}^{n-1} \sum_{j=i+1}^{n} O\left[\gamma_{i, x_{i}}, \gamma_{j, x_{j}}\right] .
$$

The edge energy integrates the edge indicator function $g$ along paths normalized by their euclidean length:

$$
E_{\text {edge }}[\Gamma]=\sum_{i=1}^{n} \frac{1}{\left|\gamma_{i, x_{i}}\right|} \int_{0}^{1} g\left(\gamma_{i, x_{i}}(u)\right)\left\|\gamma_{i, x_{i}}{ }^{\prime}(u)\right\| \mathrm{d} u .
$$

Unlike the previous edge term in Eq. (9), normalization by euclidean length is performed on each path before summation. This makes $E_{\text {edge }}$ a separable sum of path-wise terms, which is an advantageous property for optimization (this point is further discussed in subsection 3.3). As the current curve to be optimized is closed, we propose to use a region term, similar to (10), which combines image color variances of 
the two regions delimited by the curve, as proposed by (Chan and Vese, 2001):

$$
\begin{aligned}
E_{\text {region }}[\Gamma]= & \frac{\lambda}{\left|\Omega_{\text {in }}\right|} \int_{\Omega_{\text {in }}}\left\|I(\mathbf{x})-\mu_{\text {in }}\right\|^{2} \mathrm{~d} \mathbf{x} \\
& +\frac{1-\lambda}{\left|\Omega_{\text {out }}\right|} \int_{\Omega_{\text {out }}}\left\|I(\mathbf{x})-\mu_{\text {out }}\right\|^{2} \mathrm{~d} \mathbf{x},
\end{aligned}
$$

where $\lambda \in[0,1]$ controls the blending of the two terms. While the overlap and the edge energy functionals constitute the building blocks of the proposed model, the region term can be easily replaced in specific situations, e.g. with piecewise-smooth models (Lankton and Tannenbaum, 2008; Brox and Cremers, 2009) or texture features (Sagiv et al., 2006).

\subsection{Optimization}

The best sequence of labels $\left\{x_{1}, x_{2}, \ldots, x_{n}\right\}$ is determined using a brute force search among the $K^{n}$ possible configurations. Note that all energy terms are fully or partially precomputed before testing these configurations. Trivially, the edge term needs to be computed only once for each path. Overlap coefficients $O[\cdot, \cdot]$ are pre-computed between all pairs of path and stored in an upper triangular similarity matrix, allowing straightforward computation of the overlap term.

Regarding the region term, Green's theorem enables to convert region integrals over $\Omega_{\text {in }}(\Gamma)$ - and simultaneously over $\Omega_{\text {out }}(\Gamma)=\mathcal{D} \backslash \Omega_{\text {in }}(\Gamma)$ - into a sum of contour integrals over each path, according to the following template formula. For any integrable function $f$ over $\mathcal{D}$, we have:

$$
\int_{\Omega_{\mathrm{in}}(\Gamma)} f(\mathbf{x}) \mathrm{d} \mathbf{x}=\sum_{i=1}^{n} \int_{0}^{1} \mathbf{F}\left(\gamma_{i, x_{i}}\right) \cdot \gamma_{i, x_{i}}{ }^{\perp} \mathrm{d} u
$$

where $\mathbf{F}$ is a vector field verifying $\operatorname{div} \mathbf{F}=f$. The computation of color means and variances is therefore separable over each path, which allows precomputation. Vector field $\mathbf{F}$ is obtained by integrating $f$ along the $x$ and $y$-dimensions:

$$
\mathbf{F}(x, y)=\left[\int_{0}^{x} f(t, y) \mathrm{d} t, \int_{0}^{y} f(x, t) \mathrm{d} t\right]^{T}
$$

Two vector fields are computed once at every location, for $f=I$ and $f=\|I\|^{2}$, which allows color means and variances, over inner and outer regions of a given tested configuration $\Gamma$, to be efficiently determined.

\section{Experiments and discussion}

We demonstrate the ability of the model to recover closed boundaries of objects in natural color images, given few user-provided points. These points are either located on the target boundary, to assess the relevancy of the proposed approach independently from any deformation algorithm, or far from the boundary, in order to show its benefits when integrated into the deformation process of the geodesically linked active contour model. In all experiments, regularization weight $w$ was set to 0.01 , the RGB components being assumed to vary from 0 to 1 . Low values of $w$ prevent paths from creating undesirable shortcuts, therefore favouring high gradient edges. Reported execution times were obtained with a $\mathrm{C}++$ implementation running on a standard Intel Core 2 Duo $2.8 \mathrm{GHz}$ architecture with $4 \mathrm{~Gb}$ RAM.

\subsection{From points localized on the contour}

Fig. 5 depicts an experiment intended to demonstrate the consistency of contour extraction with respect to various initial locations of vertices. The closed contour was generated given $n=2$ vertices and $K=5$ admissible paths. On the aforementioned architecture, paths generation took $0.94 \mathrm{~s}$ and contour selection took $0.14 \mathrm{~s}$. As neighboring areas are sufficiently contrasted, the edge map alone turned out to be reliable enough, which allowed not to use the region homogeneity term. Hence, only the overlap and edge terms were used on this particular image. The proposed approach proves to recover suitable contours regardless of the positions of endpoints. In particular, vertices do not need to be evenly spaced along the actual object boundary.

One of the main benefits of the proposed approach over classical minimal path-based segmentation is the ability to formulate a region-based criterion, as in classical active contours. Figures 6 and 7 illustrate the interest of using such criterion, as well as the overlap constraint. The $700 \times 529$ data in Fig. 6 was processed with $n=3$ vertices and $K=5$ admissible paths per pair of successive vertices. Paths generation took $13.3 \mathrm{~s}$ and contour selection $3.9 \mathrm{~s}$. The annoying overlapping phenomenon yielded by the basic geodesically linked active contour is shown in Fig. 6(c). Incorporation of the overlap constraint enables to generate a closed contour, which remains nevertheless unrelevant with respect to image partition. This can be explained by the fact that various 

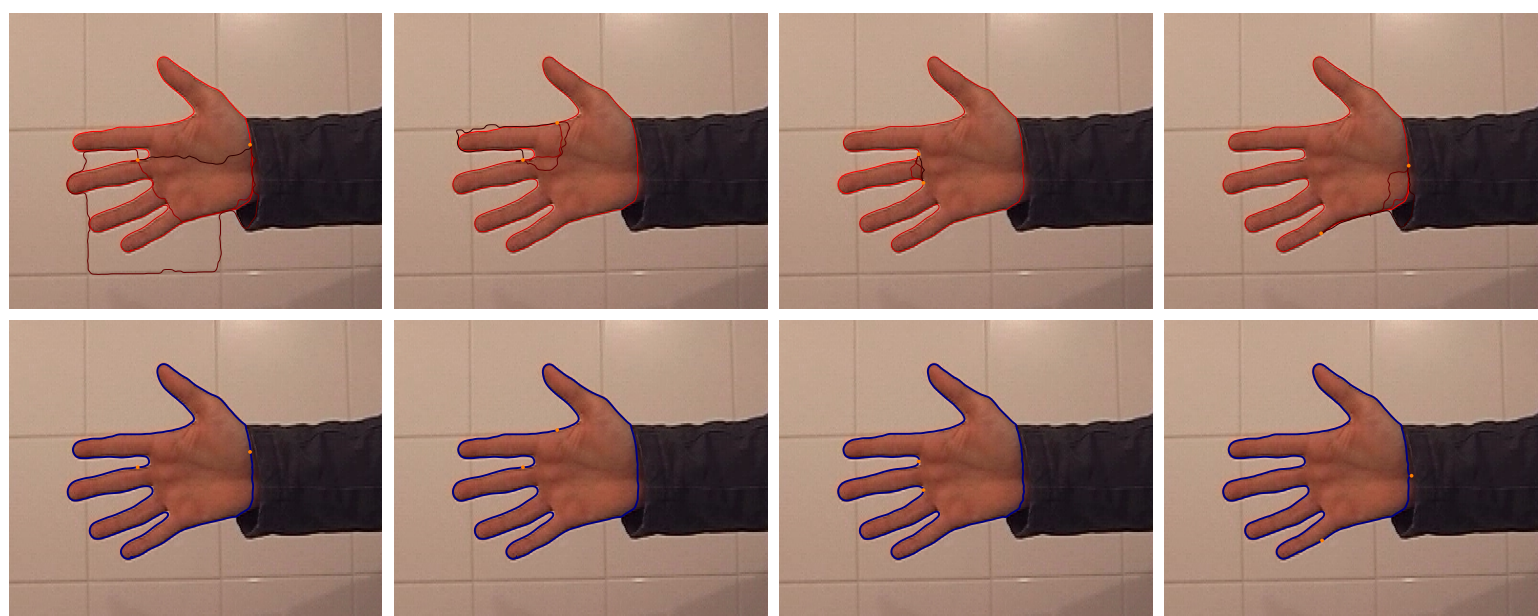

Figure 5: Robustness of contour extraction with respect to vertices locations: all admissible paths (top row) and selected contour (bottom row).

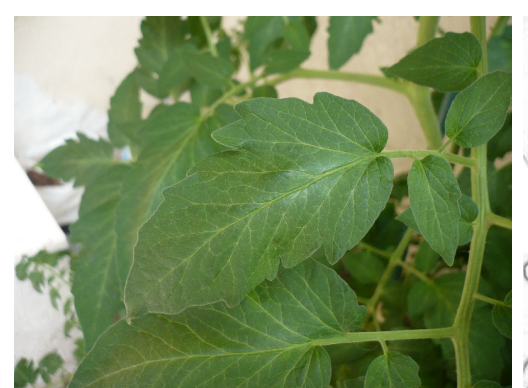

(a)

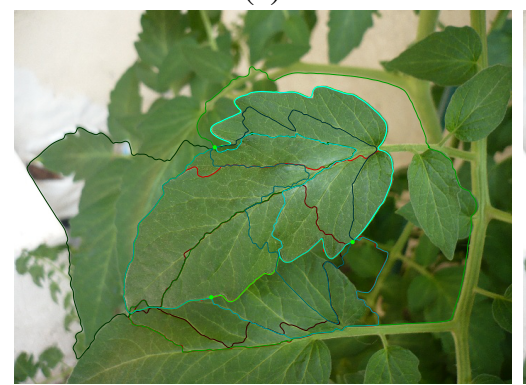

(d)

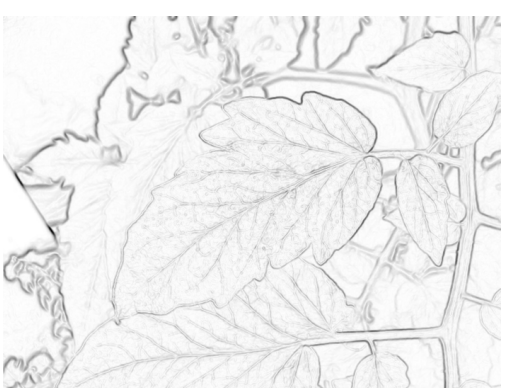

(b)

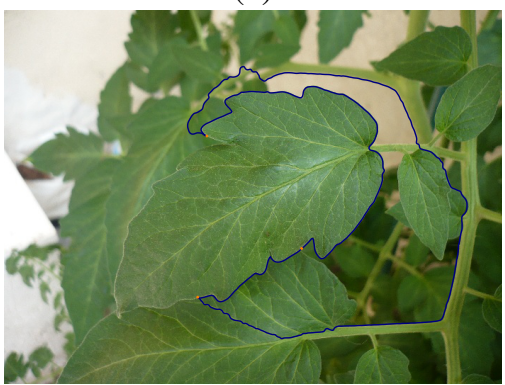

(e)

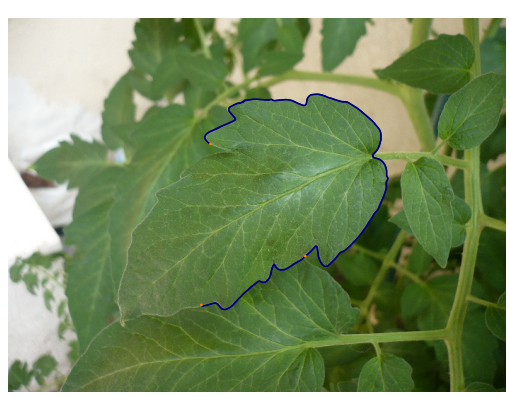

(c)

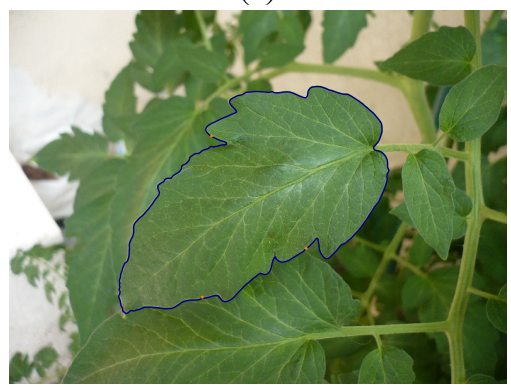

(f)

Figure 6: Influence of the region homogeneity term: (a) input image, (b) inverted gradient magnitude, (c) initial configuration of the basic geodesically linked active contour (independent minimal paths), (d) all admissible paths, (e) selected contour with overlap and edge terms, (f) selected contour with overlap, edge and region terms.

edges, stronger that the actual boundaries of the target object, can be encountered in neighboring structures. This undesirable feature is addressed by the addition of the region homogeneity term (weight $\lambda$ balancing the inner and outer region terms was set to 1 , as only the inner object is almost homogeneous). The last experiment shown in Fig. 7 follows a similar principle, with $n=4$ and $K=5$, on a $800 \times 600$ image. Reported execution times for paths generation and contour selection are $22.5 \mathrm{~s}$ and $7.8 \mathrm{~s}$, respectively.
For this image, while the proposed approach outperforms the geodesically linked active contour model at its initialization, the resulting curve fails to fully extract the desired contour, due to weak edges near the object center. Since the object of interest is textured, this could be improved by considering a region energy that favors homogeneous textured regions. 


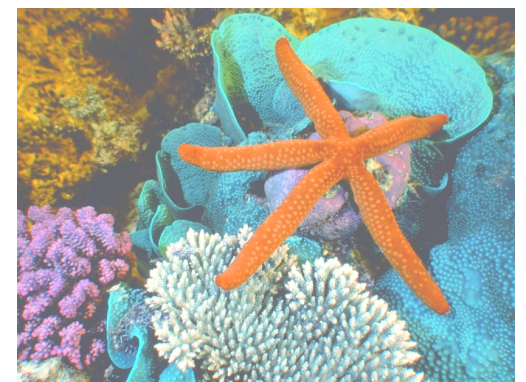

(a)

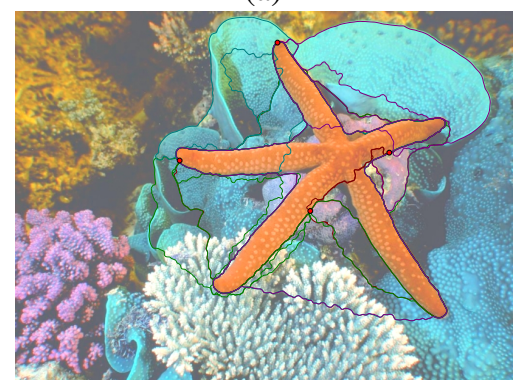

(d)

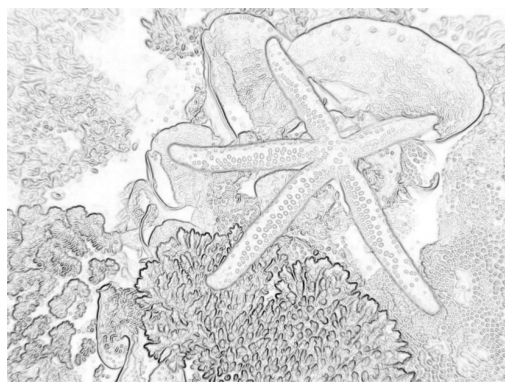

(b)

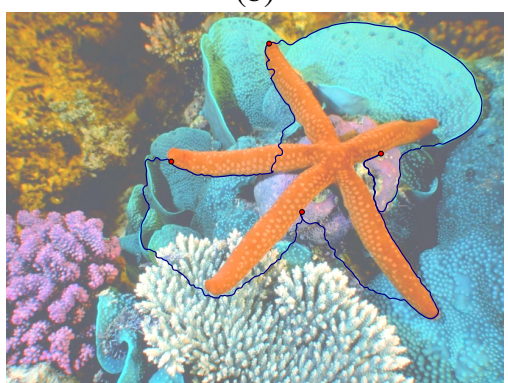

(e)

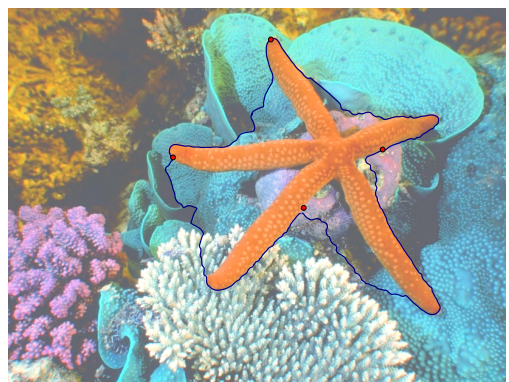

(c)

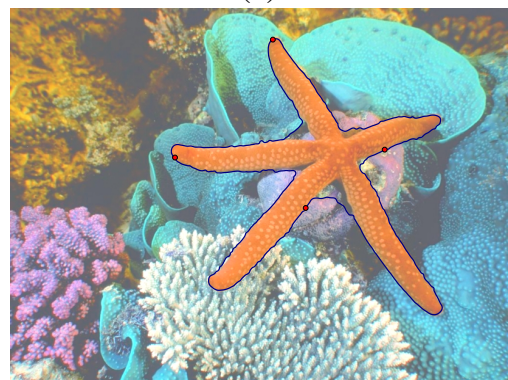

(f)

Figure 7: Influence of the region homogeneity term: (a) input image, (b) inverted gradient magnitude, (c) initial configuration of the basic geodesically linked active contour (independent minimal paths), (d) all admissible paths, (e) selected contour with overlap and edge terms, (f) selected contour with overlap, edge and region terms

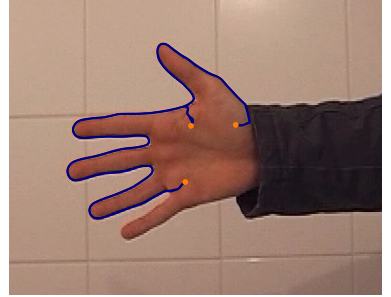

(a1)

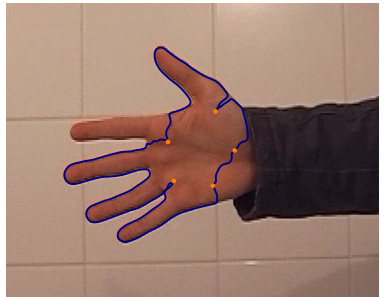

(c1)

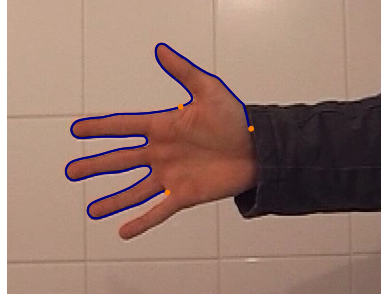

(a2)

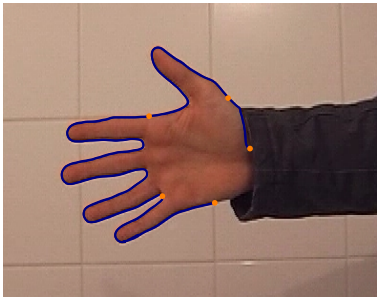

(c2)

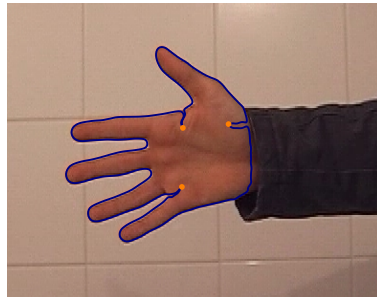

(b1)

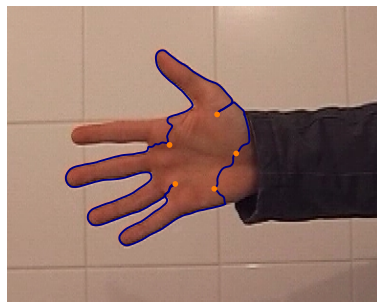

(d1)

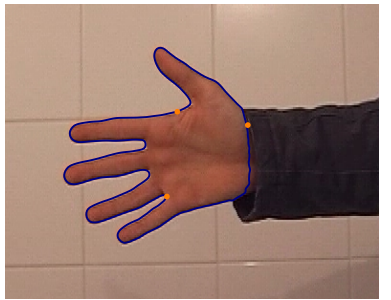

(b2)

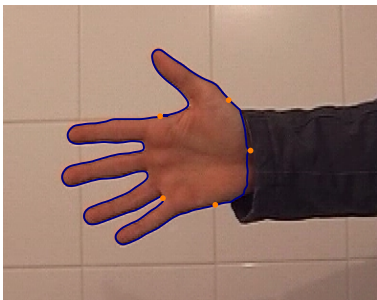

(d2)

Figure 8: Integration of non-overlapping constraint in the geodesically linked active contour model. For each subfigure: (x1) initial state, (x2) final state. (a \& c) Basic geodesically linked active contour, (b \& d) Geodesically linked active contour model with non-overlapping constraint

\subsection{Integration into deformation process}

We report experiments where the proposed approach was integrated into the evolution algorithm of the geodesically linked active contour model, so that con- tour extraction can be performed with initial points located far from the target contour. The integration is as follows: during deformation, when displacing a given vertex $\mathbf{v}_{i}$, tested geodesics $\tilde{\gamma}_{i-1}$ and $\tilde{\gamma}_{i}$ are built such that they do not overlap with existing geodesics $\left\{\gamma_{1}, \ldots, \gamma_{i-2}, \gamma_{i+1}, \ldots, \gamma_{n}\right\}$. This is achieved by deleting these existing geodesics in the potential 
map, as described in subsection 3.1. The energy of the tested contour is endowed with the overlap term according to Eq. (13).

The proposed approach can solve the overlapping problem arising in two different cases. The first case, shown in Fig. 8(a), corresponds to an overlapping present as soon as the contour is initialized and propagated afterwards. On the other hand, the second case, depicted in Fig. 8(c), shows the result of an overlapping occuring during evolution of a well-initialized curve. In both cases, the integration of our approach when updating geodesics during evolution allows to maintain a valid contour (Fig. 8(b) and Fig. 8(d)), at the expense of additional time cost to check paths configurations.

\section{Conclusion and perspectives}

By searching the best paths configurations among sets of admissible paths, given an energy functional combining an edge fitting term, a region homogeneity term and a novel overlap-penalizing energy, we aimed at overcoming some important shortcomings arising in geodesic-based segmentation. The introduced constraints allowed to guarantee consistent closed contours, whether given initial points were located on the target boundaries or far from them. Incorporation into the geodesically linked active contour model demonstrated the advantages of the approach. Future work may focus on designing finer search methods to determine the optimal set of paths, since a basic brute force search was implemented so far. A related possible investigation deals with the generation of admissible paths. In this extent, instead of generating all admissible paths per pair of successive vertices at initialization, one could think of an adaptive approach in which only necessary extra admissible paths would be created during the search process.

\section{REFERENCES}

Amini, A., Weymouth, T., and Jain, R. (1990). Using dynamic programming for solving variational problems in vision. IEEE Transactions on Pattern Analysis and Machine Intelligence, 12(9):855-867.

Benmansour, F. and Cohen, L. (2009). Fast object segmentation by growing minimal paths from a single point on 2D or 3D images. Journal of Mathematical Imaging and Vision, 33(2):209-221.

Benmansour, F. and Cohen, L. (2011). Tubular structure segmentation based on minimal path method and anisotropic enhancement. International Journal of Computer Vision, 92(2):192-210.
Brox, T. and Cremers, D. (2009). On local region models and a statistical interpretation of the piecewise smooth Mumford-Shah functional. International Journal of Computer Vision, 84(2):184-193.

Chan, T. and Vese, L. (2001). Active contours without edges. IEEE Transactions on Image Processing, 10(2):266-277.

Cohen, L. (1991). On active contour models and balloons. Computer Vision, Graphics, and Image Processing: Image Understanding, 53(2):211-218.

Cohen, L. and Kimmel, R. (1997). Global minimum for active contour models: a minimal path approach. International Journal of Computer Vision, 24(1):57-78.

Crandall, M., Ishii, H., and Lions, P.-L. (1992). User's guide to viscosity solutions of second order partial differential equations. Bull. Amer. Math. Soc., 27:1-67.

Dubuisson, M.-P. and Jain, A. (1994). A modified Hausdorff distance for object matching. In $12^{\text {th }}$ International Conference on Pattern Recognition (ICPR), pages 566-568, Jerusalem, Israel.

Eppstein, D. (1998). Finding the $k$ shortest paths. SIAM Journal of Computing, 28(2):652-673.

Kaul, V., Tsai, Y., and Yezzi, A. (2010). Detection of curves with unknown endpoints using minimal path techniques. In British Machine Vision Conference (BMVC), pages 1-12, Aberystwyth, UK.

Lankton, S. and Tannenbaum, A. (2008). Localizing regionbased active contours. IEEE Transactions on Image Processing, 17(11):2029-2039.

Mille, J. (2009). Narrow band region-based active contours and surfaces for 2D and 3D segmentation. Computer Vision and Image Understanding, 113(9):946-965.

Mille, J. and Cohen, L. (2009). Geodesically linked active contours: evolution strategy based on minimal paths. In $2^{\text {nd }}$ International Conference on Scale Space and Variational Methods in Computer Vision (SSVM), volume 5567 of $L N C S$, pages 163-174, Voss, Norway. Springer.

Peyré, G., Pechaud, M., Keriven, R., and Cohen, L. (2010). Geodesic methods in computer vision and graphics. Foundations and Trends in Computer Graphics and Vision, 5(3-4):197-397.

Sagiv, C., Sochen, N., and Zeevi, Y. (2006). Integrated active contours for texture segmentation. IEEE Transactions on Image Processing, 15(6):1633-1646.

Sethian, J. (1996). A fast marching level set method for monotonically advancing fronts. Proceedings of the National Academy of Science, 93(4):1591-1595.

Sethian, J. (1999). Level Sets Methods and Fast Marching Methods. Cambridge University Press, 2nd edition.

Tsitsiklis, J. (1995). Efficient algorithms for globally optimal trajectories. IEEE Transactions on Automatic Control, 40(9):1528-1538.

Williams, D. and Shah, M. (1992). A fast algorithm for active contours and curvature estimation. Computer Vision, Graphics, and Image Processing: Image Understanding, 55(1):14-26.

Yen, J. (1971). Finding the K shortest loopless paths in a network. Management Science, 17(11):712-716. 